Wilfrid Laurier University

Scholars Commons@ Laurier

Criminology

Laurier Brantford

$8-1-2002$

\title{
Using the Internet for Organizational Research: A Study of Cynicism in the Workplace
}

Judy Eaton

Wilfrid Laurier University, jeaton@wlu.ca

C. Ward Struthers

York University

Follow this and additional works at: http://scholars.wlu.ca/brantford_cc

\section{Recommended Citation}

Eaton, Judy and Struthers, C. Ward, "Using the Internet for Organizational Research: A Study of Cynicism in the Workplace" (2002). Criminology. 2.

http://scholars.wlu.ca/brantford_cc/2 


\title{
Using the Internet for Organizational Research: A Study of Cynicism in the Workplace
}

\author{
JUDY EATON, M.A., and C. WARD STRUTHERS, Ph.D.
}

\begin{abstract}
The Internet can be a valuable data collection tool for organizational psychology researchers. It can be less expensive than traditional paper-and-pencil survey methods, and the potential pool of participants is much larger. In addition, it can be used in situations where traditional data collection methods are not feasible, such as research involving sensitive issues such as negative employee attitudes or deviant behaviors at work. In this study, we examined the organizational attitudes of employees from various companies using (a) a snowball sample, who completed a traditional paper and pencil survey $(n=135)$, and (b) a sample recruited over the Internet, who completed an on-line survey $(n=220)$. Participants in both the nonInternet and the Internet group were asked to describe a negative incident involving their company, and answer a number of questions regarding how they felt about their company and how they behaved toward their company following the negative event. They also completed measures of organizational cynicism and job satisfaction. The two groups were compared on demographic characteristics and on their attitudes toward their organization. There were very few demographic differences between the two groups. The Internet group tended to be more cynical and to judge their organization more harshly than the non-Internet group; however, the response patterns of both groups were similar. These results suggest that, when used with caution, the Internet can be a viable method of conducting organizational research.
\end{abstract}

\section{INTRODUCTION}

$\mathbf{T}$ HE STUDY OF INDIVIDUALS IN THE WORKPLACE can present a number of challenges for organizational researchers. It can be difficult to gain access to participants, and if access is granted, there may be limits on how much time they are able to spend on the study, especially if they are completing it at work. In addition, there are certain issues about which organizations may be particularly sensitive, such as the negative effects of downsizing, deviant employee behaviors, and negative em- ployee attitudes, thus making it less likely that they will grant permission to survey their employees. If an organization does grant permission, researchers need to be alert to possible attempts by the organization to influence the research process. Organizational research can also be time-consuming and costly, and can require significant resources for data collection and coding, especially if qualitative methods are used. Traditionally, organizational researchers have relied to a great extent on case studies, employee surveys, and structured interviews in order to learn about employees

Department of Psychology, York University, Toronto, Canada. 
within organizations. Using the Internet for data collection is a new and potentially useful addition to these methods.

The purpose of this study was to assess the viability of conducting organizational research over the Internet. Data were collected from two groups of participants: one using a traditional paper-and-pencil survey and the other through an on-line Internet survey. In order to determine whether both data collection methods yielded similar results, the groups were compared on demographic characteristics, psychometric properties of the measurement instruments used, and response patterns.

The study was part of a larger study, which examined employees' feelings of cynicism toward their workplace. Employee cynicism is a growing concern among organizations, ${ }^{1}$ but because it involves negative employee attitudes and can also involve negative or deviant behavioral outcomes, organizations and their employees are often reluctant to participate in this type of research. By recruiting participants through the Internet and ensuring their anonymity, we were able to access a wide range of employed individuals from a number of different organizations.

\section{What is Internet research?}

There are a number of ways that Internet research can be conducted, although in its basic form it involves recruiting participants over the Internet (e.g., through messages posted to newsgroups, links on Web pages, directed e-mails, inclusion in search engines) and directing them to a Website that contains the study. Participants respond directly on-line, and their responses are transmitted to the researcher either through e-mail or through data files maintained by their Web server.

A number of different types of Web-based studies can be found at the American Psychological Society (APS) Website. The most common types are nonexperimental studies, in which participants read a series of questions or statements and indicate their agreement by clicking on one of a number of response options (e.g., where $1=$ strongly disagree, and $5=$ strongly agree). This information is then used to examine relationships between variables.
For example, in an I/O study on the information technology industry, ${ }^{2}$ respondents are asked a number of questions about their job search strategies, satisfaction with various aspects of their job, and the social support they receive from family and friends. Simple experimental studies can also be conducted on the Internet. For example, in one current study on personnel selection, ${ }^{3}$ participants are presented with photos and performance information about three applicants and then asked to select which one they think will be best for the position. The photos are randomly varied between participants, which enables the researchers to have two or more experimental groups.

\section{Potential benefits to organizational researchers}

In 1994, there were an estimated 22 million people with access to the Internet ${ }^{4}$; by 1995 , the estimate had risen to 30 million $^{5}$; and by 2000 , the number had risen to approximately 300 million. ${ }^{6}$ Clearly, the potential for research participants is well beyond that which could be reached with traditional survey methods. This increased pool of participants has many positive implications for organizational researchers. Firstly, it can provide access to employed individuals from a huge number of different industries and organizational settings, ${ }^{7}$ which can improve the external reliability (i.e., generalizability) of a study. For some organizational issues, studying a single organization may not provide enough variability. For example, if a particular organization had, through downsizing, lack of communication, or some other event that was perceived as negative and unjustified by employees, created a culture of cynicism in its employees, ${ }^{8}$ studying that particular organization would not provide much information about patterns of cynicism. Studying organizational cynicism across a wide variety of industries, companies, and job positions, however, is more conducive to developing and testing a model of cynicism that can be applied regardless of the corporate culture.

Secondly, the larger, and hence wider, participant pool available through the Internet 
can facilitate cross-cultural organizational research. 5,9 Although most Internet users are in North America, there are an estimated 69 million people with Internet access in Asia/ Pacific and 83 million in Europe. ${ }^{6}$

Finally, it is also easier to reach more specialized populations such as those working in particular industries or job positions, gays/ lesbians/bisexuals, and people with physical disabilities or medical conditions. $4,10,11$ If a researcher was interested, for example, in how people with physical disabilities cope with workplace stress, posting a message to a newsgroup for people with disabilities could reach more potential participants than soliciting them directly from specific organizations.

In addition to the benefits of a wider participant pool, Internet research is also both time- and cost-effective. ${ }^{5}$ Although there are equipment and labor costs associated with developing the study and posting it on the Internet, once the study is on-line, the involvement of the researchers should be minimal. Costs can be reduced through the elimination of paper-based testing materials, postage, and often staffing to run the study and enter data. The results of the study can also be e-mailed to participants or posted on a Web page, thus further reducing postage costs. Internet research is also more environmentally responsible, in that less paper is used. Intrepid researchers can also use any one of a number of Web survey-development programs to create the on-line version of their study themselves, eliminating the labor costs of hiring a professional Web developer. This can be a timeconsuming process, however, and the value of the expertise and creative talents of an experienced Web survey developer should not be underestimated.

Web-based studies can also provide more anonymity for participants. ${ }^{4}$ Responses that are sent from the participant's computer by e-mail to the researcher will not include the participant's name or e-mail address (the "From" line is left blank). Unless the participant voluntarily includes it, no identifying information about his or her organization is sent to the researcher. This is especially important for organizational research that involves assessing employee attitudes and/or behaviors, because employees may be reluctant to admit to having negative thoughts or feelings toward their job or organization in a company-sanctioned study. As long as a sufficient amount of demographic and job-related information is collected from participants, it should not be necessary for researchers to know the name of the organization or the employee. The benefits to participants of this added confidentiality and assurance of anonymity will usually outweigh the costs to researchers of not having this information.

\section{Limitations of Internet organizational research}

There are limitations to conducting organizational research over the Internet. One of the main problems is the lack of control over the test setting. ${ }^{4,5}$ In a Web-based study, participants complete the study on their own time, often on their own computer in their own home. Given that the researcher is not present, it is impossible to control for extraneous variables such as noise, time of day, respondent motivation, and even equipment differences such as the type and speed of computer and modem. It should be noted, however, that most of the same concerns apply to paper-andpencil surveys that employees fill out on their own time. Nevertheless, for this reason, Internet studies are not appropriate for research that requires accurate timing, interpersonal interactions, or color perception, or for longitudinal or high-impact designs. 4

In addition to internal validity issues, external validity can also be a problem. Participants are self-selected, making generalization to non-computer-literate Internet users difficult because the sample is not random. We would argue, however, that very little organizational research uses random samples, and because published studies often involve participants from a single organization, generalizability is problematic throughout traditional organizational research.

\section{Validity of data collected on the Internet}

Studies that have been conducted to test whether data collected over the Internet is 
equivalent to traditional data collection methods, although varying in quality, tend to find support in favor of Internet research. In one of the few specifically organizational studies conducted on the Internet, Stanton 7 found similarities in item variability and internal covariance patterns between a pencil-and-paper sample and an Internet sample. Nonorganizational research that has also found evidence of equivalency between pencil-and-paper samples and Internet samples includes studies of personality, $^{12}$ human sexuality, ${ }^{4,13}$ interpersonal attractiveness, 10,14 decision-making, 15 and pictorial communication. ${ }^{16}$ Although it is clear that certain types of research are not feasible to conduct over the Internet, the above list of successful Internet studies, which is far from exhaustive, suggests that there are many instances where Internet research can be an appropriate data collection tool.

The focus of the present study was on organizational phenomena. Evidence of the practicality of the Internet for social and personality psychology, although encouraging, does not necessarily generalize to workplace issues. Employed individuals may make up a significant portion of all Internet users; nevertheless, they comprise a subgroup of users, and hence it is important to determine whether the Internet is appropriate for targeting this subgroup.

\section{The present study}

The purpose of the study was to test the viability of using the Internet to recruit and collect data from participants for an organizational study. Two sets of data were collected: one set from a group of employed individuals who were recruited using a snowball sample and tested using a traditional pencil-andpaper survey; and one set from a group of employed individuals who were recruited over the Internet and tested using an on-line survey posted on the Internet. The two samples were compared on demographic characteristics, the psychometric properties of the measures used, and general response patterns. Given that this is an exploratory study, specific hypotheses were not generated. It was expected, however, based on existing studies comparing Internet and non-Internet samples, that the two samples would be similar.

\section{MATERIALS AND METHODS}

\section{Participants}

The pencil-and-paper group consisted of 135 employed individuals from a wide variety of organizations. They were recruited using a snowball technique, whereby students in an upper-level Organizational Psychology undergraduate class at a Canadian university were asked to return two surveys completed by employed family members, friends, or coworkers in return for course credit. Surveys were returned to the researchers in sealed envelopes. Participants were entered into a draw to win one of two $\$ 50$ prizes.

The Internet sample consisted of $220 \mathrm{em}-$ ployed individuals from a wide variety of organizations. Participants were recruited via the Internet to complete a study on workplace attitudes. Links to the study were included on Web-based lists of on-line psychological studies, Internet search engines, and a number of Web pages (a complete list can be obtained from the authors). Survey responses were transmitted to the researchers via e-mail. No identifying information was included on the e-mail form, and participants were assured of anonymity and confidentiality. They were offered no compensation for their participation, other than the opportunity to receive a copy of the results when the study was completed.

\section{Measures}

This study was part of a larger study on organizational cynicism. The purpose of the larger study was to test a social motivational theory of cynicism, in which individuals are expected to become cynical toward their organization following negative events that are perceived by the employee as being controllable by the organization and stable (i.e., likely to happen again in the future). For the purposes of the current study, only the four main variables from the larger study were used. 
1. Critical incident report. Participants were asked to write a few sentences about a negative incident involving their organization.

2. Response to negative event. Participants were asked a number of questions relating to the negative incident, including how responsible the organization was for the incident, how angry and how sympathetic they felt toward the organization, and to what extent they engaged in negative behaviors or wanted to engage in negative behaviors toward the organization following the event (where $1=$ not at all, and 7 = a great deal) .

\section{Organizational cynicism. Organizational} cynicism was measured using the Organizational Cynicism Scale, ${ }^{17}$ a 10 -item scale that measures aspects of cynicism about one's workplace. It includes such items as "I believe that my company lacks integrity" (where $1=$ strongly disagree, and $5=$ strongly agree). Cronbach's alpha for the scale is 0.90 .

4. Job satisfaction. Job satisfaction was measured with a three-item Job Satisfaction Scale developed for the purpose of this study. It includes items such as "I am satisfied with my job" (where 1 = strongly disagree, and 5 = strongly agree). Cronbach's alpha for the scale is 0.83 .

\section{Procedure}

The study was called "The Workplace Attitudes Study" and was described as a study to examine how people feel about negative events that happen in their workplace. Participants in the non-Internet sample were approached by a student in the class to complete the survey. The package they were given contained an informed consent form, the survey, and a ballot for the $\$ 50$ draws. The survey contained the measures described above and some demographic questions. Once completed, participants were asked to place the survey in the envelope and seal it, following which the student returned it to the researchers.

Participants in the Internet sample accessed the study by either clicking on a link from another web page or by typing the Internet ad- dress of the study into their Web browser. The first page of the study contained a short description of the study, and participants were instructed to click a button if they were interested in taking part. Those who continued with the study were asked to read an informed consent form and to click a button labeled "I Agree" if they understood it and agreed to participate in the study. They were informed that they could stop the study at any time by simply leaving the website, and that their answers were anonymous and could not be traced back to them.

Once they clicked the "I Agree" button, participants were led to a web page containing the questionnaire described above and the same demographic variables as the non-Internet sample. Participants could scroll up or down and change their answers at any point. Once finished, they were instructed to click a button labeled "Submit" to complete the study. A page then appeared that thanked them for their participation, explained the study in more detail, and provided an e-mail address from which they could request more information and study results.

\section{RESULTS}

The samples were first compared on demographic characteristics to determine if there were any significant differences between the Internet and non-Internet groups (Table 1). With the exception of the gender composition of the groups, independent-samples $t$ tests showed no significant differences between the groups. The groups were remarkably similar in age. Participants in both groups worked similar numbers of hours per week and had been working at their company for an equivalent length of time. They also had approximately the same number of years of work experience. Participants were also similar in the types of jobs they held. The most frequent type of job for both groups was sales/service/customer support. The majority of the remaining participants categorized their jobs as either professional, office/clerical, or education (Table 1). Of some concern with this variable is that two 
TABlE 1. COMPARISON OF NON-INTERNET AND INTERNET SAMPLE DEMOGRAPHICS

\begin{tabular}{lcc}
\hline Variable & Non-Internet & Internet \\
\hline$n$ & 135 & 220 \\
Age & 31.8 & 31.3 \\
Gender (\% male) & $48 \%$ & $29 \%$ \\
Hours worked/week & 38 & 37 \\
Years at company & 5 & 4.6 \\
Work experience (years) & 11.6 & 12.5 \\
Job position & & \\
Sales & $29 \%$ & $25 \%$ \\
Professional & $13 \%$ & $18 \%$ \\
$\quad$ Office/clerical & $12 \%$ & $14 \%$ \\
$\quad$ Education & $7 \%$ & $13 \%$ \\
Computer & $7 \%$ & $7 \%$ \\
Self-employed & $5 \%$ & $1 \%$ \\
Other & $27 \%$ & $22 \%$ \\
\hline
\end{tabular}

out of $135(1.5 \%)$ of the non-Internet participants did not complete the question on job type, whereas 35 out of $220(16 \%)$ of the Internet participants left this question blank.

The groups differed significantly in terms of gender composition, with the non-Internet group having relatively equal numbers of males and female (48\% male) and the Internet group comprising only $29 \%$ males $(t=3.75$; $p<0.001)$. In our non-Internet sample, we requested that students recruit one male and one female participant, if possible, which resulted in a relatively balanced sample. With the Internet sample, no such control was possible.

We also examined the factor structure and psychometric properties of the measures used. Factor analysis of the Organizational Cynicism and Job Satisfaction Scales showed similar factor structures for each scale in both groups. In both the non-Internet and the Internet groups, all 10 items of the Organizational Cynicism Scale loaded onto a single factor, with factor loadings between 0.58 and 0.84 for the nonInternet group and between 0.47 and 0.82 for the Internet group. Similarly, all three items of the Job Satisfaction Scale loaded onto a single factor, with factor loadings between 0.82 and 0.93 for the non-Internet group and between 0.78 and 0.90 for the Internet group.

Internal reliability of both scales were also equivalent across samples. In the non-Internet group, the Organizational Cynicism Scale had a Cronbach's alpha of 0.91, and in the Internet group, alpha was 0.90 . For the Job Satisfaction
TABLE 2. COMPARISONS OF NON-INTERNET AND INTERNET SAMPLES ON MAIN STUDY VARIABLES

\begin{tabular}{lrrrr}
\hline & \multicolumn{2}{c}{ Means } & & \\
\cline { 2 - 3 } Variable & $\begin{array}{c}\text { Non- } \\
\text { Internet }\end{array}$ & Internet & \multicolumn{1}{c}{$t$} & $p$ \\
\hline Responsibility & 5.50 & 5.92 & 2.40 & 0.017 \\
Anger & 4.98 & 5.48 & 2.82 & 0.005 \\
Sympathy & 2.83 & 2.33 & -2.65 & 0.009 \\
Job satisfaction & 9.68 & 8.43 & -3.45 & 0.001 \\
Cynicism & 26.90 & 34.14 & 6.63 & 0.000 \\
Negative behavior & 3.08 & 3.69 & 2.96 & 0.003 \\
Negative intentions & 3.56 & 4.64 & 4.72 & 0.000 \\
\hline
\end{tabular}

Scale, alpha was 0.86 in the non-Internet group and 0.81 in the Internet group.

In order to examine differences in the response patterns of the two groups, independent-samples $t$ tests were performed on each of the main variables. All of the comparisons were significant, indicating that the mean responses to these variables differed significantly between the Internet and non-Internet group (Table 2). Figure 1 shows the pattern of responses for each group. An examination of this graph suggests that, although the means of the two groups differed, their response patterns were very similar. Participants in the Internet group were clearly more harsh in their judgments of their organization, with higher scores on responsibility (the level of responsibility they believe the organization has for the event), anger, cynicism, and negative behaviors and behavioral intentions toward the organization, and lower scores on sympathy and job satisfaction.

\section{DISCUSSION}

The results of this study provide some evidence for the viability of conducting organizational research over the Internet. Demographically similar samples were obtained, although gender composition varied between the two groups. The psychometric properties of the measures used were encouragingly similar for both groups, suggesting that the Internet might be an ideal place to conduct initial scale development studies. Participants' responses to the main variables differed significantly between groups. Upon closer analysis, 
Response patterns

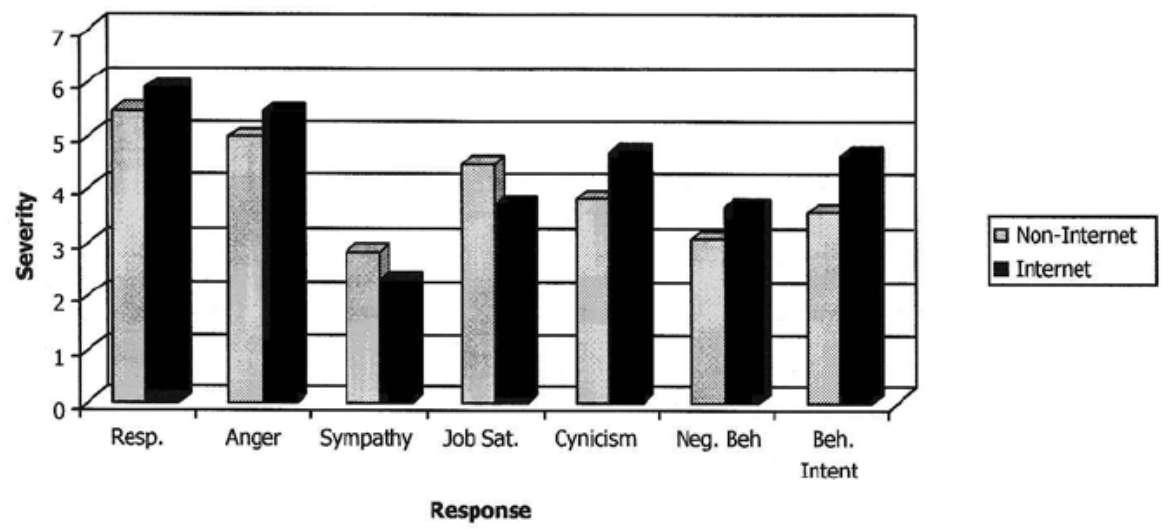

FIGURE 1 Comparison of response patterns of non-internet and internet groups. Note: For ease of comparison, Job Satisfaction and Cynicism have been converted to 7-point scales.

however, it became evident that although the means were different, the patterns of responses were similar, with the Internet sample providing more severe or harsh responses.

The similarity in response patterns suggests that the difference between the non-Internet and Internet samples is one of degree, in that although the participants in the Internet sample were more negative in their ratings of their organization, they were consistently more negative in their ratings of similar variables. It is possible that those in the Internet sample felt that they had more anonymity, and hence felt that they could be more candid in their responses. There is evidence that computerized assessments increase self-disclosure and candor among research participants. ${ }^{18-21}$ More recent evidence suggests that this "candor hypothesis" may also apply to Internet-based studies. ${ }^{14}$ Perhaps the participants in our Internet sample, encouraged by the anonymous nature of the submission process (with the click of a button their responses were sent directly to an unseen and unknown researcher), were more honest in reporting their judgments of their organization. The non-Internet sample may have been deterred from being as honest, because they had to return their completed questionnaire, albeit in a sealed envelope, to the student, who would then return it to the researcher. There is some support for this hypothesis in our study. We asked participants to write a short paragraph regarding a negative incident involving them and their organization. Although a qualitative analysis of their responses is beyond the scope of this paper, in general, the descriptions from the Internet participants tended to be longer and more detailed than those in the nonInternet group. This suggests that self-disclosure and candor was increased in the Internet group.

An alternative explanation for the differences between groups is that those in the Internet sample actually did have more negative feelings toward their organization. This could have occurred as a result of self-selection bias in the Internet group. All participants were aware that their participation was voluntary, but because those in the non-Internet group were approached by someone they knew, and were offered a monetary incentive for participating (the chance to win one of two $\$ 50$ draws), their reasons for taking part in the study may have been different than those in the Internet group, who were not approached directly, and who were not given a monetary incentive. It is possible that those in the Internet group who participated did so because the study gave them the opportunity to express negative feelings about their organization; those who did not feel negatively may not have been motivated to participate, thus resulting in a negatively biased sample. The non-Internet sample may have felt more pressure to participate for other reasons, such as wanting to help their friend, or wanting to win the money, and hence participated regardless 
of how they felt about their organization. This could have resulted in a more balanced sample in the non-Internet group, at least in terms of how they feel about their organization. Future research in this area should attempt to reduce selection bias by ensuring that similar recruiting methods are used for each group.

The only other significant difference between groups was in the gender composition. Relatively equal numbers of males and females were expected in the non-Internet group, because we requested that each student recruit one male and one female participant. In the Internet group, however, we had no such control. In a comparison of the demographic characteristics of participants from Internet studies between 1986 and 1999, Krantz and Dalal$^{22}$ found, with some exceptions, steadily increasing numbers of female participants (from 16\% in 1986 to as high as 56\% in 1999). Our $71 \%$ female sample may be an extension of this growing trend in female participants. It could also be evidence that women are more willing to complete an Internet survey. A Webbased initiative to link Web researchers with participants by maintaining a database of participants who are willing to participate in survey research over the Internet lists its current sample as comprising $69 \%$ females. ${ }^{23}$

The high incidence of missing data in the job type variable may be a result of the increased candor in the Internet group. If they responded with more candor, and hence more negatively, then perhaps they were more concerned about being linked to their organization. Although participants were ensured complete anonymity, it is possible that some of them felt uncomfortable providing this information after they had disclosed negative feelings about their organization. Another possible explanation is that the missing data is due to computer hardware or software issues. For example, if that particular question appeared at the bottom of the computer screen on some users' machines, it may have been inadvertently missed. Extensive pilot testing is crucial for a successful Internet study; however, it is impossible to test the survey on every potential type of computer that respondents may use to complete the survey. In order to determine if there were systematic differ- ences between those in the Internet group who identified their job type and those who did not, post hoc analyses of these participants were performed. Comparisons of the ratings of the two groups on the dependent variables were not significant, indicating that the two groups did not differ in their feelings toward their organization after the negative event. A comparison of the demographic characteristics of these two groups showed that those who did not report their job type tended to be older (35.6 years of age) than those who did report their job type (30.5 years of age) and, not surprisingly, had more overall work experience (17 as opposed to 11.7 years). There was no difference in the length of time they had worked for their present company. This finding suggests that older workers may be more cautious about providing too much identifying information on the Internet, although they appear to respond with similar amounts of candor.

Overall, the results of this study suggest that the Internet can be a promising addition to the data collection tools used by organizational researchers. The fact that the responses of participants recruited and surveyed over the Internet were similar to those of participants tested using traditional organizational methods provides evidence for the equivalence of the two methods. Demographically, the samples were similar, although organizational researchers should expect higher percentages of female participants in Internet studies that are not targeted at specific populations. Our results also suggest that some Internet participants (particularly more mature participants) may not be convinced of the complete anonymity of this type of research; indeed, the field would benefit from improved ways of ensuring absolute anonymity to Internet respondents.

Based on these findings, we would recommend Internet data collection methods to organizational researchers who are interested in conducting pilot studies, scale development research, and other types of exploratory work. The fact that most of our participants complied with our request to write a paragraph describing a negative event at work (in fact, we were pleasantly surprised at their candor in this regard) suggests that simple qualitative studies could also be conducted successfully over the 
Internet. Future research should address the potential problems of self-selection bias, ensuring respondent anonymity, and gender differences. We believe, however, that the future of Internet research and data collection is promising, and that organizational researchers could benefit from using this technique to supplement their existing research methods.

\section{ACKNOWLEDGMENTS}

We would like to thank Sue Waldeck for her invaluable assistance with Web programming. This research was supported in part by an Ontario Graduate Scholarship to the first author and a Social Sciences and Humanities Research Counsel (SSHRC) Standard Research Grant (no. 410-98-1016) to the second author.

\section{REFERENCES}

1. Kanter, D.L., \& Mirvis, P.H. (1989). The cynical Americans. San Francisco: Jossey-Bass.

2. Lin, L.F. (2001). Information technology survey [On-line]. Available: http://personal.bgsu.edu / lflin/itworkforcesurveymb.htm (12/10/01).

3. Bauer, C., \& Lagankie, J. (2001). Personnel selection study. [On-line]. Available: http://141.217.109.238 / number2.htm (12/10/01).

4. Smith, M.A., \& Leigh, B. (1997). Virtual subjects: using the Internet as an alternative source of subjects and research environment. Behavior Research Methods, Instruments, $\mathcal{E}$ Computers 29:496-505.

5. Michalak, E.E., \& Szabo, A. (1998). Guidelines for Internet research: an update. European Psychologist 3:70-75.

6. Nancarrow, C., Pallister, J., \& Brace, I. (2001). A new research medium, new research populations and seven deadly sins for Internet researchers. Qualitative Market Research: An International Journal 4:136-149.

7. Stanton, J. (1998). An empirical assessment of data collection using the Internet. Personnel Psychology 51:709-725.

8. Eaton, J., \& Struthers, C.W. (2001, June). A social motivation approach to organizational cynicism. Presented at the Canadian Psychological Association Conference, Ste-Foy, QC.

9. Barry, D.T. (2001). Assessing culture via the Internet: methods and techniques for psychological research. CyberPsychology and Behavior 4:17-21.

10. Epstein, J., Klinkenberg, W.D., Wiley, D., et al. (2001). Insuring sample equivalence across internet and paper-and-pencil assessments. Computers in Human Behavior 17:339-346.

11. McKenna, K.Y., \& Bargh, J.A. (1998). Coming out in the age of the Internet: identity "demarginalization: through virtual group participation. Journal of Personality and Social Psychology 75:681-694.

12. Buchanan, T., \& Smith, J. (1999). Using the Internet for psychological research: personality testing on the World Wide Web. British Journal of Psychology 90: 125-144.

13. Bailey, R.D., Foote, W.E., \& Throckmorton, B. (2000). Human sexual behavior: a comparison of college and Internet surveys. In: Birnbaum, M.H., ed. Psychological experiments on the Internet. San Diego, CA: Academic Press, pp. 141-168.

14. Buchanan, T. (2000). Internet research: self-monitoring and judgments of attractiveness. Behavior Research Methods, Instruments, $\mathcal{E}$ Computers 32:521-527.

15. Birnbaum, M.H. (2000). Decision making in the lab and on the Web. In: Birnbaum, M.H., ed. Psychological experiments on the Internet. San Diego: Academic Press, pp. 3-34.

16. Pagani, D., \& Lombardi, L. (2000). An intercultural examination of facial features communicating surprise. In: Birnbaum, M.H., ed. Psychological Experiments on the Internet. San Diego: Academic Press, pp. 169-194.

17. Eaton, J., \& Struthers, C.W. (2001). The development of the Organizational Cynicism Scale (unpublished data).

18. Carr, A.C., \& Ghosh, A. (1983). Accuracy of behavioral assessment by computer. British Journal of Psychiatry 142:66-70.

19. Evan, W.M., \& Miller, J.R. (1969). Differential effects on response bias of computer vs. conventional administration of a social science questionnaire: an exploratory methodological experiment. Behavioral Science 14:216-227.

20. Levine, S., Ancill, R.J., \& Roberts, A.P. (1988). Assessment of suicide risk by computer-delivered selfrating questionnaire: preliminary findings. Acta Psychiatrica Scandinavica 80:216-220.

21. Rosenfeld, P., Giacalone, R.A., Knouse, S.B., et al. (1991). Impression management, candor and microcomputer-based organizational surveys: an individual differences approach. Computers in Human Behavior 7:23-32.

22. Krantz, J.H., \& Dalal, R. (2000). Validity of Webbased psychological research. In: Birnbaum, M.H., ed. Psychological experiments on the Internet. San Diego: Academic Press, pp. 35-60.

23. Stanton, J., \& Weiss, E. (2001). Sample characteristics [On-line]. Available: www.psychology.gatech.edu / eweiss/sample.htm.

Address reprint requests to: Judy Eaton, M.A. Department of Psychology Behavioural Sciences Building, Rm. 205 York University 4700 Keele St. Toronto, ON, M3J 1P3, Canada E-mail: jeaton@yorku.ca 